\title{
Increased Gene Expression of the Cardiac Endothelin System in Obese Mice
}

\author{
Authors \\ Affiliations \\ R. A. Catar ${ }^{1,2, *}$, G. Muller ${ }^{1,3,}$ *, A. Brandt ${ }^{1,4}$, H. Langbein ${ }^{1}$, C. Brunssen ${ }^{1}$, C. Goettsch ${ }^{1,5,6}$, A. Frenzel $^{1}$, \\ A. Hofmann ${ }^{1}$, W. Goettsch ${ }^{1}$, N. Steinbronn ${ }^{4}$, R. H. Strasser ${ }^{4}$, U. Schubert ${ }^{7}$, B. Ludwig ${ }^{7}$, S. R. Bornstein ${ }^{7}$, \\ H. Morawietz \\ Affiliation addresses are listed at the end of the article
}

\author{
Key words \\ - atherosclerosis \\ - body weight \\ - cholesterol \\ - LDL cholesterol \\ obesity
}

received 17.06 .2014

accepted 05.08.2014

Bibliography
DOI http://dx.doi.org/
10.1055/s-0034-1387761
Published online:
September 2, 2014
Horm Metab Res 2015;
47: 509-515
@ Georg Thieme Verlag KG
Stuttgart · New York
ISSN 0018-5043
Correspondence
H. Morawietz, PhD
Department of Medicine III
Division of Vascular Endothe-
lium and Microcirculation
University Hospital Carl Gustav
Carus
TU Dresden
Fetscherstraße 74
01307 Dresden
Germany
Tel.: + 49/351/458 6625
Fax: + 49/351/458 63 54
Henning.Morawietz@
tu-dresden.de

Bibliography

DOI http://dx.doi.org/

10.1055/s-0034-1387761

Published online:

September 2, 2014

Horm Metab Res 2015;

47: 509-515

Stuttgart · New York

ISSN 0018-5043

Correspondence

Division of Vascular Endothe-

lium and Microcirculation

University Hospital Carl Gustav

Carus

Fetscherstraße 74

01307 Dresden

Germany

Fax: + 49/351/458 6354

tu-dresden.de

\section{Abstract \\ $\nabla$}

Obesity is a well-known risk factor of atherosclerosis and heart failure. In the human heart, a local endothelin system containing prepro-endothelin-1, endothelin-converting enzyme-1, and endothelin receptors A and B has been described. The endothelin system is activated in heart failure; however, the impact of obesity on the cardiac endothelin system is unknown. In this study, 18-week-old male C57BL/6 mice fed either a control diet or a high-fat diet for 10 weeks were analyzed. High-fat diet significantly increased the body weight of the animals and augmented lowdensity lipoprotein, high-density lipoprotein, and cholesterol plasma levels, compared to control. The animal groups showed no significant differences in left ventricular size or function (heart

\section{Introduction}

\section{$\nabla$}

Obesity, in addition to insulin resistance, hyperlipidemia, and hypertension, is a hallmark of the metabolic syndrome leading to type 2 diabetes and increasing the risk of cardiovascular disease [1]. Several cardiovascular risk factors are known to affect the endothelin system. Increased endothelin-1 (ET-1) synthesis is considered to be involved in the development and progression of heart failure [2]. In addition, circulating levels and cardiac tissue levels of ET- 1 are upregulated in experimental models of heart failure and can predict the clinical outcome in cardiovascular diseases [3].

ET-1 synthesis includes proteolytic cleavage of precursor peptide big ET-1 by the metalloprotease endothelin-converting enzyme-1 (ECE-1). Inhibition of ECE-1 activity has been suggested as a therapeutic strategy in the treatment of cardiovascular diseases [4]. ET-1 binds to endothelin

* Both first authors contributed equally to this study. rate, ejection fraction, fractional shortening, left ventricular posterior wall thickness, cardiac output) after control or high-fat diet. We did not observe signs of cardiac hypertrophy or changes in markers of cardiac fibrosis in these heart samples. The cardiac expression of prepro-endothelin-1 mRNA, endothelin-converting enzyme-1 mRNA, and protein and endothelin receptors $A$ and $B$ mRNA was increased in 18-week-old obese C57BL/6 mice compared to animals with normal weight $(\mathrm{p}<0.05$ vs. control). Furthermore, endothelin-1 plasma levels showed an increasing trend. In conclusion, an increased expression of genes of the endothelin system was observed in the hearts of 18-week-old mice after high-fat diet, possibly contributing to later cardiovascular complications of obesity.

receptors $A$ and $B\left(E_{A}, E_{B}\right)$, thus mediating the regulation of vascular tone, positive inotropic effects, and cardiac hypertrophy. Local cardiac ET-1 synthesis has been shown in endocardium, myocardium, and coronary endothelium. Determinants of ET- 1 biosynthesis are selectively regulated within this myocardial compartment in heart failure. Furthermore, both $\mathrm{ET}_{\mathrm{A}}$ and $\mathrm{ET}_{\mathrm{B}}$ receptors are expressed in murine and human myocardium. However, the impact of obesity on the cardiac endothelin system is currently unknown. Therefore, the aim of this study was to analyze the impact of high-fat diet on cardiac function and the endothelin system in obese mice.

\section{Materials and Methods v Animals}

Eight-week-old male C57BL/6 ( $\mathrm{n} \geq 7$ /group) mice were fed control diet EF R/M CD88137 (as control) or high-fat diet EF R/M TD88137 (containing 
21 \% butter fat; Ssniff Spezialdiäten GmbH, Soest, Germany) for 10 weeks. The procedure was carried out in accordance with the "Guide for the Care and Use of Laboratory Animals" published in 1996 by the National Academy Press and approved by the institutional ethic commission for animal experiments of the Medical Faculty of the University of Technology Dresden and the government of Saxony (AZ: 24-9168.11-1/2010-24).

\section{Echocardiography}

In a separate set of experiments, cardiac function was analyzed by echocardiography in 18-week-old male C57BL/6 mice fed a control diet or high-fat diet for 10 weeks ( $n=12 /$ group). Echocardiography was performed using a VisualSonics Vevo 770 system (VisualSonics, Toronto, Ontario, Canada) with a 707B $(30 \mathrm{MHz})$ scanhead. Mice were anesthetized with $3 \%$ isoflurane and then placed in a supine position on a temperature-controlled operating table. ECG electrodes were connected to the limbs. A rectal probe to record animal temperature was applied and chest hair was subsequently removed by depilatory cream. Isoflurane concentration was set to $2 \%$ and maintained at this level during the measurements. Ultrasound gel was applied and the left ventricle analyzed. B- and M-Mode pictures and loops were recorded, first in parasternal long view and afterwards in parasternal short axis view. Stroke volume and cardiac output (CO) were automatically calculated in parasternal long axis view. Ejection fraction (EF), fractional shortening (FS) and left ventricular posterior wall thickness (LVPWD) were recorded and automatically calculated from parasternal short axis view.

\section{Blood glucose and serum lipid measurements}

Blood glucose and serum lipid measurements were performed after fasting overnight. Blood glucose was measured in peripheral blood from tail tip using the OneTouch Ultra system (LifeScan, Neckargemünd, Germany). All lipid measurements in murine blood serum were performed at the Institute of Clinical Chemistry and Laboratory Medicine at the Medical Faculty of the University of Technology Dresden using a Roche/Hitachi MODULAR analyzer and kits for triglycerides (TG), cholesterol (Chol), high-density lipoprotein cholesterol (HDL-C plus 2nd generation) and low-density lipoprotein cholesterol (LDL-C plus $2^{\text {nd }}$ generation), were all purchased from Roche Diagnostics GmbH, Mannheim, Germany.

\section{Histology}

Heart paraffin sections were generated as previously described [5]. In brief, after 18 weeks including 10 weeks of control diet or high-fat diet, hearts were rapidly excised and fixed in buffered formalin $(4 \%)$, followed by rinsing in PBS $(4 \times 1 \mathrm{~h})$, dehydration and paraffin embedding. Serial $5 \mu \mathrm{m}$ sections were cut, deparaffinized and rehydrated through degraded ethanol. Heart structure was stained by hematoxylin and eosin (HE). Collagen content was analyzed by picrosirius red staining [6].

\section{RNA isolation and quantification of mRNA expression}

At 18 weeks of age, hearts were excised for RNA and protein isolation. Cardiac biopsies of animals were dissected immediately after explantation, snap-frozen and stored in liquid nitrogen until RNA preparation. Total RNA from myocardial tissue was isolated using the TRIFAST protocol (Peqlab, Erlangen, Germany). RNA concentration was determined by UV spectrophotometry. The mRNA expression of components of the cardiac endothelin system (prepro-endothelin-1 or ppET-1, ECE-1, $\mathrm{ET}_{\mathrm{A}}$ and $\mathrm{ET}_{\mathrm{B}}$ ) was quantified by quantitative reverse transcriptionpolymerase chain reaction (RT-PCR) and normalized to $18 \mathrm{~S}$ rRNA in the RNA from murine hearts. The following primer were used: ppET-1 sense ( $5^{\prime}$-AAC TCA GGG CCC AAA GTA CC-3'), antisense (5'-TTT GCA ACA CGA AAA GAT GC-3') (annealing temperature: $60^{\circ} \mathrm{C}, 30$ cycles, fragment size: $169 \mathrm{bp}$ ); ECE- 1 sense (5'-CAT GGA CCC CAC AGT AGA CC-3'), antisense (5'-CTC CCA GCT TCT CAA TCA GC-3') (annealing temperature: $66^{\circ} \mathrm{C}, 30$ cycles, fragment size: $281 \mathrm{bp}$ ); $\mathrm{ET}_{\mathrm{A}}$ sense (5'-TGC ACG GCT ATT GCC CAC AG-3'), antisense (5'-AGA GGG AAC CAG CAC AGG GC-3') (annealing temperature: $68^{\circ} \mathrm{C}, 30$ cycles, fragment size: 772 bp); $\mathrm{ET}_{\mathrm{B}}$ sense (5'-CGA GCT GTT GCT TCT TGG AGT C-3'), antisense ( $5^{\prime}$-CCG GAA GTT GTC ATA TCC GTG ATC-3') (annealing temperature: $69^{\circ} \mathrm{C}, 30$ cycles, fragment size: $\left.702 \mathrm{bp}\right)$; and $18 \mathrm{~S}$ rRNA sense ( $5^{\prime}$-GTT GGT GGA GCG ATT TGT CTG G -3'), antisense (5'-AGG GCA GGG ACT TAA TCA ACG C-3') (annealing temperature: $60^{\circ} \mathrm{C}, 11$ cycles, fragment size: $346 \mathrm{bp}$ ).

In additional experiments, mRNA expression of genes involved in cardiac fibrosis was quantified by real-time PCR in the Applied Biosystems 7500 Fast Real-Time PCR system (Applied Biosystems, Foster City, CA, USA). Specific oligonucleotide primers were synthesized by TIB MolBiol SyntheseLabor (Berlin, Germany). The reactions were carried out in $20 \mu$ reaction volumes containing corresponding cDNA, gene specific primers $(0.5 \mu \mathrm{M}$ each) and Power SYBR Green PCR Master Mix (Applied Biosystems, Foster City, CA, USA). Primer sequences: collagen, type I, alpha 1 (Col1a1) sense (5'-GAG CGG AGA GTA CTG GAT CG-3'), antisense (5'-GTT CGG GCT GAT GTA CCA GT-3'); collagen, type III, alpha 1 (Col3a1) sense (5'-GCA CAG CAG TCC AAC GTA G-3'), antisense (5'-TCT CCA AAT GGG ATC TCT GG-3'); and $\beta-2$ microglobulin (B2m) sense (5'-GAA ATC CAA ATG CTG AAG AAC G-3'), antisense (5'-CAA ATG AAT CTT CAG AGC ATC ATG-3'). Reaction conditions included a 2-min initial activation step at $50^{\circ} \mathrm{C}$ and a 10 -min denaturing step at $95^{\circ} \mathrm{C}$, followed by 40 cycles of $15 \mathrm{~s}$ at $95^{\circ} \mathrm{C}$, and $1 \mathrm{~min}$ at $60^{\circ} \mathrm{C}$. Specificity of the reaction was verified by performing melting curve analysis at the end of each series of assays. The relative amount of the transcript was calculated by the cycle threshold method using the Applied Biosystems 7500 System v.1.2.3 software and normalized to the endogenous reference gene (B2m).

\section{Protein isolation and Western blot analysis}

Protein from myocardial tissue was isolated using the TRIFAST protocol. The protein concentration was determined with Pierce BCA Protein Assay Kit (Thermo Fisher Scientific Inc., Waltham, MA, USA).

Equal amounts of protein were separated by SDS-PAGE (10\%) and transferred to nitrocellulose membranes (Schleicher \& Schuell, Dassel, Germany). Membranes were incubated with polyclonal antibodies generated against ECE-1, ET $\mathrm{A}, \mathrm{ET}_{\mathrm{B}}$ (Santa Cruz Biotechnology, Inc., Santa Cruz, CA, USA) and secondary horseradish peroxidase-linked anti-rabbit IgG (GE Healthcare, Freiburg, Germany). The protein expression was detected with Western Lightning Chemiluminescence Reagent Plus (PerkinElmer Life Sciences, Boston, MA, USA) and quantified using AIDA Image Analyzer software (Raytest, Berlin, Germany). The protein expression was normalized to glyceraldehyde-3-phosphodehydrogenase (GAPDH) using a monoclonal antibody (HyTest, Turku, Finland) and a secondary horseradish peroxidase-linked anti-mouse IgG (GE Healthcare, Freiburg, Germany). 

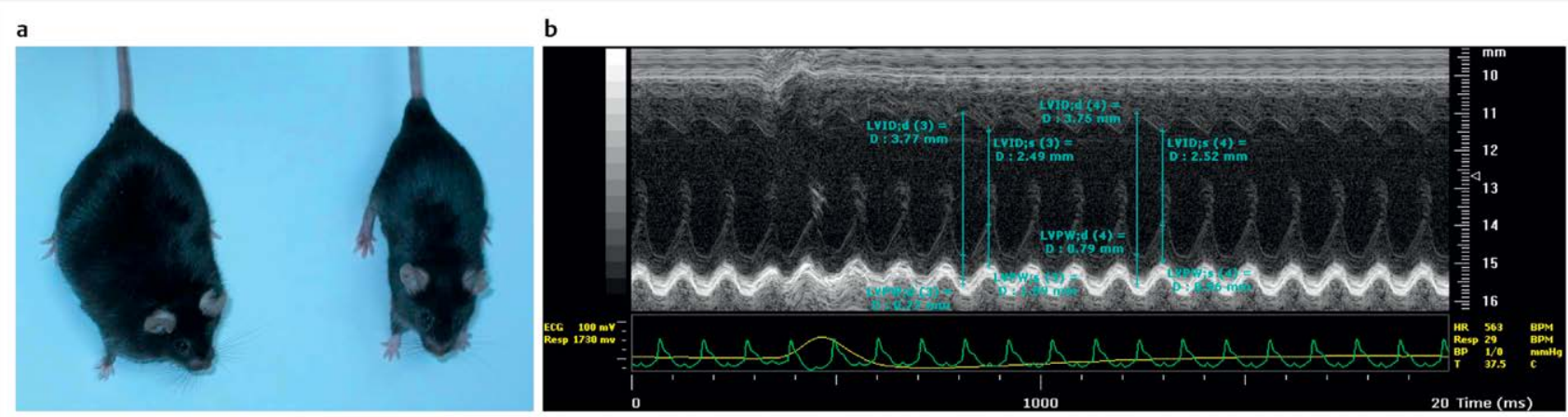

Fig. 1 Phenotype and echocardiography. a Phenotype of 18-week-old mice after 10 weeks of high-fat diet. Right: 18 -week-old C57BL/6 mice fed with standard chow, left: typical 18-week-old sibling after 10 weeks of high-fat diet. b A typical M-Mode picture of the left ventricle of a C57BL/6 mouse (control diet) was taken from left apical 2-chamber view. Recordings of the electrocardiogram are displayed in green and of the respiratory curve in yellow. Recorded values and reading points are indicated in blue. BP: Blood pressure; ECG: Electrocardiogram; HR: Heart rate; LVID;d: Left ventricular inner diameter in diastole; LVID;s: Left ventricular inner diameter in systole; LVPW;d: Left ventricular posterior wall thickness in diastole; LVPW;s: Left ventricular posterior wall thickness in systole; Resp: Respiration (signal in $\mathrm{mV}$ indicated on the left; ratemeter result in BPM displayed on the right); T: Body temperature.

\section{Endothelin-1 plasma levels}

Endothelin levels were quantified in plasma of 18 -week-old mice fed a control diet or high-fat diet for 10 weeks using the Endothelin-1 Quantikine ELISA Kit (R \& D Systems, Inc., Minneapolis, MN, USA).

\section{Statistical analysis}

Data are shown as mean \pm SEM. Statistical analysis was performed with Student's $t$-test (SigmaStat, Systat Software, Erkrath, Germany). A p-value of $<0.05$ was considered to be statistically significant.

\section{Results \\ $\nabla$}

\section{Lipid parameters}

After receiving a high-fat diet for 10 weeks, the body weight of the animals was significantly increased from $24.5 \pm 0.6 \mathrm{~g}$ of the control diet group to $34.2 \pm 1.9 \mathrm{~g}$ of the high-fat diet group $(\mathrm{p}<0.05$ vs. control) ( $\odot$ Fig. 1a). This was accompanied by augmented blood glucose, low-density lipoprotein and cholesterol plasma levels in the obese animals. These data and additional parameters are summarized in $\bullet$ Table 1.

\section{Cardiac function}

In a separate set of experiments, cardiac function was analyzed by echocardiography in 18-week-old male C57BL/6 mice fed a control diet or high-fat diet for 10 weeks ( $n=12 /$ group). A typical M-Mode picture of the left ventricle of a $\mathrm{C} 57 \mathrm{BL} / 6$ mouse (control diet) taken from left apical 2-chamber view is shown in $\triangle$ Fig. 1b. The echocardiographic data are summarized in $\odot$ Table 2 . The animal groups showed no significant differences in left ventricular size or function (heart rate, ejection fraction, fractional shortening, left ventricular posterior wall thickness, cardiac output) after 10 weeks of control diet or high-fat diet (18 weeks of age). We did not observe signs of heart failure in these animals.

\section{Cardiac structure and fibrosis}

In an independent set of experiments, we analyzed the size and structure (by HE staining) and the collagen content (by picrosirius red staining, collagen in red on a pale yellow background) of
Table 1 Body weight and blood parameters of C57BL/6 mice.

\begin{tabular}{lcc} 
Parameter & \multicolumn{2}{c}{ C57BL/6 } \\
& Control diet & High-fat diet \\
\hline Body weight $(\mathrm{g})$ & $24.5 \pm 0.6$ & $34.2 \pm 1.9^{*}$ \\
Blood glucose $(\mathrm{mM})$ & $10.0 \pm 0.2$ & $12.5 \pm 1.4^{*}$ \\
\hline Triglycerides $(\mathrm{mM})$ & $1.3 \pm 0.1$ & $1.3 \pm 0.1$ \\
\hline Cholesterol $(\mathrm{mM})$ & $2.5 \pm 0.3$ & $5.4 \pm 0.4^{*}$ \\
\hline HDL-cholesterol $(\mathrm{mM})$ & $2.1 \pm 0.1$ & $4.4 \pm 0.3^{*}$ \\
LDL-cholesterol $(\mathrm{mM})$ & $0.3 \pm 0.1$ & $1.1 \pm 0.1^{*}$
\end{tabular}

${ }^{*} \mathrm{p}<0.05$ vs. control diet, mean \pm SEM. HDL: High-density lipoprotein, LDL: Lowdensity lipoprotein, SEM: Standard error of the mean

Table 2 Echocardiographic data of C57BL/6 mice.

\begin{tabular}{lcc|} 
Parameter & \multicolumn{2}{c|}{ C57BL/6 } \\
& Control diet & High-fat diet \\
\hline HR (bpm) & $462.2 \pm 81$ & $460 \pm 60$ \\
\hline EF (I. a. 2-chamber view) (\%) & $59.9 \pm 1.9$ & $65.2 \pm 2.9$ \\
\hline FS (l. a. 2-chamber view) (\%) & $31.3 \pm 1.3$ & $34.0 \pm 2.0$ \\
CO (ml/min) & $10.7 \pm 3.5$ & $11.37 \pm 3.1$ \\
\hline LVPW;d (mm) & $0.84 \pm 0.05$ & $0.86 \pm 0.11$ \\
\hline BW (g) & $23.2 \pm 2.3$ & $32.8 \pm 3.0^{*}$ \\
\hline * $P$ <0.05 vs. control diet, mean \pm SEM & & \\
HR: Heart rate; EF: Ejection fraction; FS: Fractional shortening; l. a.: Left apical; \\
LVPWD: Left ventricular posterior wall thickness; CO: Cardiac output; SEM: Standard \\
error of the mean
\end{tabular}

cardiac sections in mice fed a control diet or a high-fat diet for 10 weeks ( $\bullet$ Fig. 2). We did not observe increased signs of hypertrophy or fibrosis in these cardiac biopsies. In additional experiments, we analyzed mRNA expression of markers of cardiac fibrosis collagen, type I, alpha 1 (Col1a1) and collagen, type III, alpha 1 (Col3a1) by real-time PCR ( $\odot$ Fig. 3). The cardiac mRNA expression of Col1a1 and Col3a1 was not different in 18-weekold wild-type mice fed a control diet or high-fat diet for 10 weeks.

\section{Gene expression of endothelin system}

In obese C57BL/6 mice, cardiac expression of prepro-endothelin-1 mRNA was significantly increased compared to the control 


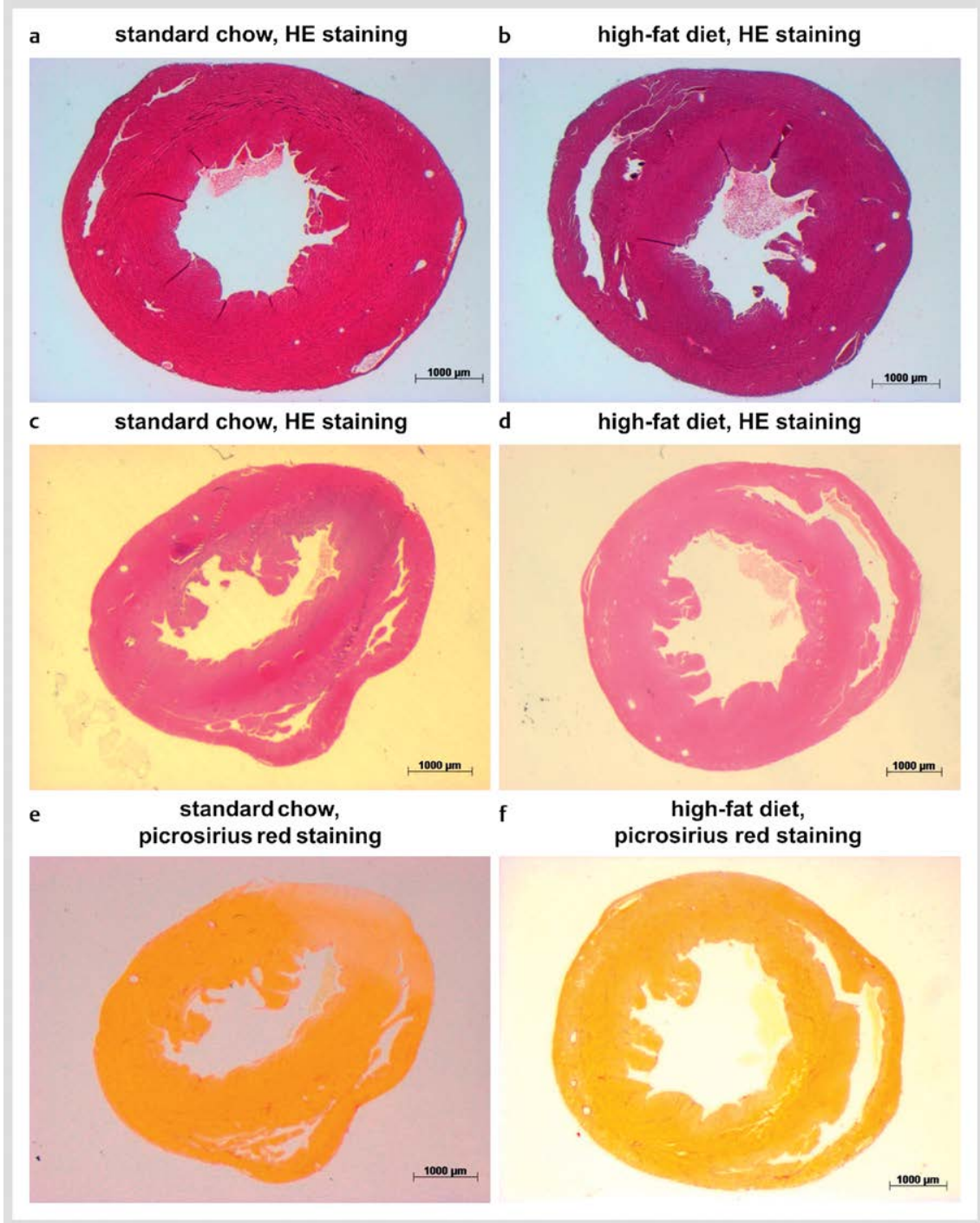

Fig. 2 Cardiac structure and collagen content. Size and structure $\mathbf{a}-\mathbf{d}$, by HE staining) and collagen content $\mathbf{e}-\mathbf{f}$, stained in red by picrosirius red staining) of representative cardiac sections in mice fed a control diet a, c, e or a high-fat diet b, d, f for 10 weeks.

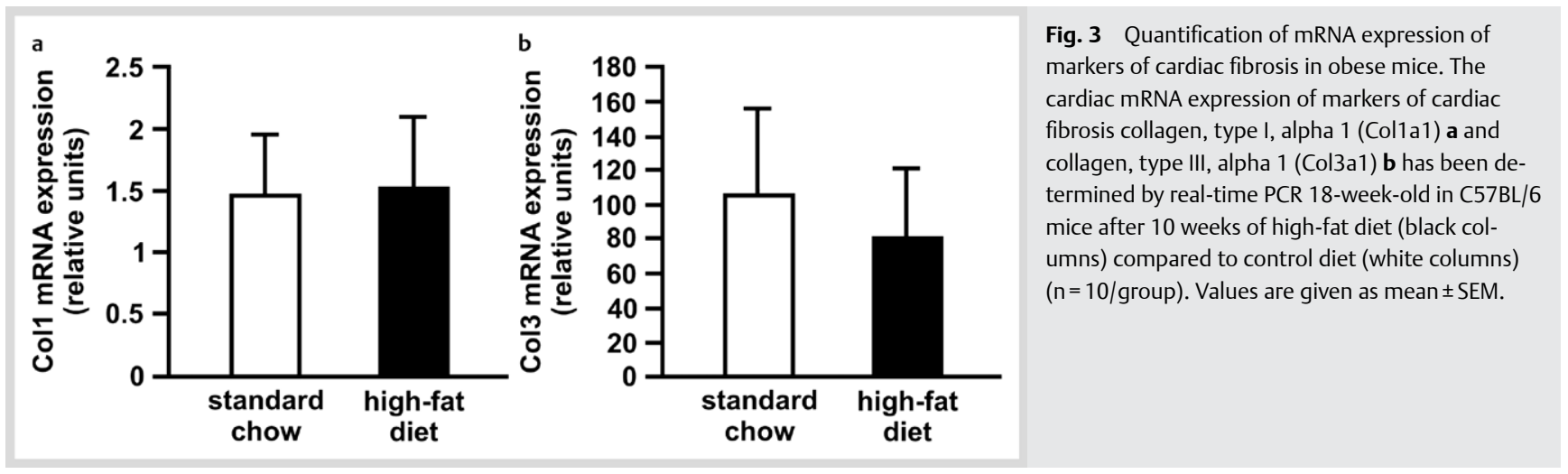

group ( $\odot$ Fig. 4a). Endothelin-1 plasma levels in 18-week-old mice fed a control diet $(n=12)$ or a high-fat diet $(n=11)$ for 10 weeks showed a trend to be increased $(124 \pm 19 \%$ of control) without reaching significance ( $\odot$ Fig. 4b). High-fat diet induced the endothelin-converting enzyme- 1 mRNA ( $\odot$ Fig. 4c) and protein ( $\odot$ Fig. 4d) expression in hearts from C57BL/6 mice compared to mice fed control diet ( $p<0.05$ vs. control).

Furthermore, mRNA expression of cardiac endothelin receptor A and $B$ was upregulated by high-fat diet in the murine heart ( $\odot$ Fig. 4e, f). The protein expression of cardiac endothelin recep- tors A (135 $\pm 56 \%$ of control) and B (158 $\pm 74 \%$ of control) in 18-week-old mice on high-fat diet showed a trend to be increased without reaching statistical significance.

\section{Discussion}

$\nabla$

Wild-type mice might not reflect all lipid parameters observed in clinical studies. The increase in total cholesterol and LDL-cho- 


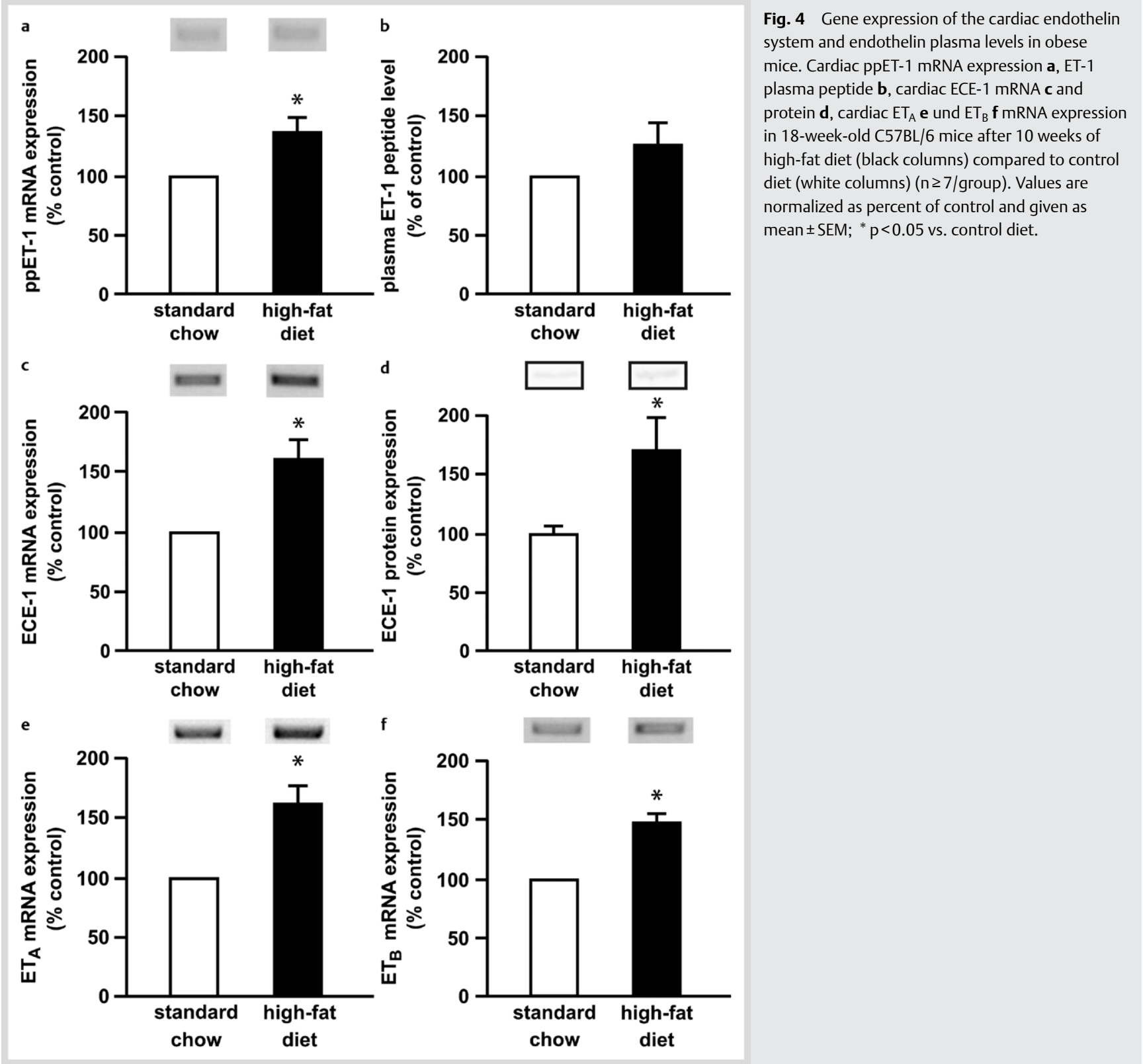

lesterol in our mice is in agreement with previous data [7]. Other studies showed increased total cholesterol and LDL-cholesterol, but no increase in plasma triglycerides in mice after high-fat diet as well $[8,9]$. Furthermore, discrepancies between different studies might have been due to metabolic and/or endocrine adaptations to the long-term high-fat diet and the different genetic backgrounds between mouse models.

The increased HDL-cholesterol levels in our mice are in agreement with previous studies feeding a Western diet to C57BL/6 mice [7]. The HDL-cholesterol is important in the lipid transport of these mice. The cholesterol content of Western diets is known to play a major role in the paradoxical increase in HDL [8]. A high-saturated fatty acid- and cholesterol-containing diet can promote the fecal excretion of macrophage- and HDL-derived cholesterol. This change in reverse cholesterol transport may constitute a compensatory mechanism to protect macrophages from cholesterol accumulation [8]. In addition, both dietary saturated fat and cholesterol intake are known to raise plasma high-density lipoprotein cholesterol (HDL-C) levels. Several epidemiological studies and 1 meta-analysis of 60 controlled trials showed a positive correlation between high saturated fat intake and HDL-C as well $[10,11]$.

The blood glucose levels in our control mice appear to be quite high after overnight fasting. C57BL/6 is the most widely used inbred strain and wild-type control. C57BL/6J mice fed a high-fat diet develop obesity and mild to moderate hyperglycemia (The Jackson Laboratory, Bar Harbour, ME, USA). Therefore, we have chosen this strain as a murine model to study some of the dietinduced changes seen in patients with metabolic syndrome. In agreement with previous findings, we observed in our mice further elevated blood glucose levels after high-fat diet. A putative explanation could be a naturally occurring deletion in nicotinamide nucleotide transhydrogenase (Nnt) exons 7-11 occurred in C57BL/6J sometime prior to 1984 . This deletion results in the absence of the NNT protein, and is associated with impaired glucose homeostasis control and reduced insulin secretion [12]. Furthermore, the type and duration of diet, duration of fasting and collection of blood samples might affect the blood glucose levels as well [13]. 
We did not observe significant changes in cardiac function at 18 weeks of age. The cardiac parameters were in the normal physiological range as described in the literature $[14,15]$. Therefore, the animals did not display functional signs of heart failure in response to high-fat diet at the time point of our study. In additional experiments, we did not find signs of cardiac hypertrophy and fibrosis in heart sections of animals from both experimental groups. Furthermore, we did not observe changes in the cardiac gene expression of collagens. They are well-established markers of cardiac fibrosis [16]. This further supports the view that both groups did not differ in cardiac structure or function.

This study demonstrates an upregulation of the mRNA expression of genes of the cardiac endothelin system in C57BL/6 mice after 10 weeks of high-fat diet. Obesity can be associated with an ET-1-dependent activation of renal angiotensin-converting enzyme and an enhanced vascular contractility to angiotensin II [17]. Furthermore, endothelin might modulate increased endothelium-dependent vasoconstriction in obesity [18]. Our findings are supported by the recently described increased serum ET-1 levels and augmented myocardial ppET- 1 mRNA expression in mice fed a high-fat diet [19]. Interestingly, this study suggested an involvement of leptin in the upregulation of ET-1. The endothelin-1 peptide plasma levels and the endothelin receptor A and B protein expression in our mice showed a trend to be increased without reaching significance. The circulating plasma ET-1 levels might rather reflect a spill-over of the vascular ET-1 production and has been used as predictor of prognosis in patients with heart failure [20]. Even while the ET-1 plasma level might not be directly linked to the local cardiac expression of components of the endothelin system, the slightly increased ET-1 level supports an activation of the endothelin synthesis in these animals. As increased plasma endothelin-1 levels were found in normotensive and hypertensive obese subjects compared with a lean healthy group [21], our findings could also have clinical implications. We could previously show that endothelin-converting enzyme-1 mRNA and ET-1 peptide levels were increased in failing myocardium of patients and partially normalized by ACE inhibition [22]. The endothelin receptor A was 2-fold higher expressed in human atrium, compared to ventricle [23]. Furthermore, endothelin receptor A was markedly upregulated in failing human myocardium and normalized by ventricular assist device unloading [24].

We could not determine whether upregulation of the endothelin system was due to augmented gene expression in cardiac myocytes or nonmyocytes (e.g. endothelial cells, smooth muscle cells, or fibroblasts). ECE-1 protein expression was in previous studies more widespread compared to its mRNA, being present in endothelial cells, mesenchymal cells and myocytes, and particularly enriched in the trabeculae and nascent ventricular conduction system [25]. The 2 endothelin receptors were found to be expressed in cardiomyocytes as well as in the cardiac vessels [26]. Ventricular ET-1 receptors have a cell-specific distribution: the majority of ET receptors in cardiomyocytes are of the $\mathrm{ET}_{\mathrm{A}}$ subtype, whereas fibroblasts had a nearly equal proportion of the $\mathrm{ET}_{\mathrm{A}}$ and $\mathrm{ET}_{\mathrm{B}}$ subtypes [27]. The ET receptor number on smooth muscles and fibroblasts does not exceed ET receptor number on myocytes. Endothelial cells express $\mathrm{ET}_{\mathrm{B}}$ receptors only. Therefore, the increased cardiac mRNA expression of the endothelin receptors most likely reflects an increased mRNA expression in cardiomyocytes.

A putative mechanism of activation of the endothelin system in obesity could involve LDL. In agreement with clinical data obe- sity increased the LDL-cholesterol level in our study as well. We could show in previous in vitro studies that native and oxidized LDL increases the ppET- 1 and ECE- 1 mRNA expression and the ET-1 peptide formation in human endothelial cells [28]. Furthermore, the endothelial $\mathrm{ET}_{\mathrm{B}}$ receptor mRNA was induced by both lipoproteins [29]; therefore, the increased LDL levels of the obese mice could contribute to the accelerated expression of genes of the endothelin system, suggesting a novel mechanism explaining how obesity supports the development of cardiac hypertrophy and heart failure. This might have also implications for the development of novel therapeutic strategies in heart failure [30]. In summary, our data show that the elevated expression of genes of the endothelin system in obese animals is independent from histological or functional signs of heart failure or cardiac fibrosis. An activated endothelin system might represent an early marker of compensatory efforts to maintain cardiac function in obesity and might contribute to later cardiovascular complications of obesity.

\section{Acknowledgements \\ $\nabla$}

We are grateful to K. Borgwald, C. Bodenstein, and J. Mittag for excellent technical assistance. This work was supported by grants from Faculty of Medicine of TU Dresden (MeDDrive program to G.M. and B.L.), BMBF (to H.M.), Doktor Robert Pfleger Foundation, Bamberg, Germany (to H.M.), Else Kröner-Fresenius-Stiftung (to H.M.), European Section of the Aldosterone Council (ESAC) Deutschland (to A.H., H.M.), Deutsche Forschungsgemeinschaft (DFG) (1695/4-1, 1695/5-1 to H.M., KFO 252 to B.L., H.M., S.R.B.), and DFG Center for Regenerative Therapies Dresden (CRTD), Cluster of Excellence (to W.G., H.M.).

\section{Conflicts of Interest}

$\nabla$

The authors declare that they have no conflict of interest that could be perceived as prejudicing the impartiality of the research reported.

Affiliations

Department of Medicine III, Division of Vascular Endothelium and

Microcirculation, University Hospital Carl Gustav Carus, TU Dresden,

Dresden, Germany

${ }^{2}$ Department of Nephrology and Medical Intensive Care, Charité-

Universitätsmedizin Berlin, Campus Virchow-Klinikum, Berlin, Germany ${ }^{3}$ GWT-TUD GmbH, Dresden, Germany

${ }^{4}$ Department of Internal Medicine and Cardiology, TU Dresden, Dresden, Germany

${ }^{5}$ Department of Medicine III, Division of Endocrinology, Diabetes, and

Metabolic Bone Diseases, University Hospital Carl Gustav Carus, TU Dresden, Dresden, Germany

${ }^{6}$ Department of Medicine, Center for Interdisciplinary Cardiovascular Sciences, Brigham and Women's Hospital, Harvard Medical School, Boston, Massachusetts, USA

Department of Medicine III, University Hospital Carl Gustav Carus,

TU Dresden, Dresden, Germany

\section{References}

1 Lamounier-Zepter V, Bornstein SR, Ehrhart-Bornstein M. Mechanisms of obesity-related hypertension. Horm Metab Res 2004; 36: 376-380

2 Masaki T. Historical review: Endothelin. Trends Pharmacol Sci 2004; 25: 219-224

3 Sakai S, Miyauchi T, Kobayashi M, Yamaguchi I, Goto K, Sugishita Y. Inhibition of myocardial endothelin pathway improves long-term survival in heart failure. Nature 1996; 384: 353-355 
4 Jeng AY. Utility of endothelin-converting enzyme inhibitors for the treatment of cardiovascular diseases. Curr Opin Invest Drugs 2003; 4: 1076-1081

5 Wunderlich C, Schober K, Kasper M, Heerwagen C, Marquetant R, Ebner $B$, Forkmann M, Schoen S, Braun-Dullaeus RC, Schmeisser A, Strasser $R H$. Nitric oxide synthases are crucially involved in the development of the severe cardiomyopathy of caveolin-1 knockout mice. Biochem Biophys Res Commun 2008; 377: 769-774

6 Whittaker P, Kloner RA, Boughner DR, Pickering JG. Quantitative assessment of myocardial collagen with picrosirius red staining and circularly polarized light. Basic Res Cardiol 1994; 89: 397-410

7 Desmarchelier C, Dahlhoff C, Keller S, Sailer M, Jahreis G, Daniel H. C57Bl/6 N mice on a western diet display reduced intestinal and hepatic cholesterol levels despite a plasma hypercholesterolemia. BMC Genomics 2012; 13: 84

8 Escola-Gil JC, Llaverias G, Julve J, Jauhiainen M, Mendez-Gonzalez J, Blanco-Vaca F. The cholesterol content of Western diets plays a major role in the paradoxical increase in high-density lipoprotein cholesterol and upregulates the macrophage reverse cholesterol transport pathway. Arterioscler Thromb Vasc Biol 2011; 31: 2493-2499

9 Kim S, Sohn I, Ahn JI, Lee KH, Lee YS, Lee YS. Hepatic gene expression profiles in a long-term high-fat diet-induced obesity mouse model. Gene 2004; 340: 99-109

10 Kotseva K, Stagmo M, De Bacquer D, De Backer G, Wood D; Group EIS. Treatment potential for cholesterol management in patients with coronary heart disease in 15 European countries: findings from the EUROASPIRE II survey. Atherosclerosis 2008; 197: 710-717

11 Mensink RP, Zock PL, Kester AD, Katan MB. Effects of dietary fatty acids and carbohydrates on the ratio of serum total to HDL cholesterol and on serum lipids and apolipoproteins: a meta-analysis of 60 controlled trials. Am J Clin Nutr 2003; 77: 1146-1155

12 Freeman HC, Hugill A, Dear NT, Ashcroft FM, Cox RD. Deletion of nicotinamide nucleotide transhydrogenase: a new quantitive trait locus accounting for glucose intolerance in C57BL/6J mice. Diabetes 2006; 55: 2153-2156

13 Ayala JE, Samuel VT, Morton GJ, Obici S, Croniger CM, Shulman GI, Wasserman DH, McGuinness OP, Consortium NIHMMPC. Standard operating procedures for describing and performing metabolic tests of glucose homeostasis in mice. Dis Model Mech 2010; 3: 525-534

14 Rottman JN, Ni G, Brown M. Echocardiographic evaluation of ventricular function in mice. Echocardiography 2007; 24: 83-89

15 Stypmann J. Doppler ultrasound in mice. Echocardiography 2007; 24: $97-112$

16 de Haas HJ, Arbustini E, Fuster V, Kramer CM, Narula J. Molecular imaging of the cardiac extracellular matrix. Circ Res 2014; 114: 903-915

17 Barton M, Carmona R, Morawietz H, d'Uscio LV, Goettsch W, Hillen H, Haudenschild CC, Krieger JE, Munter K, Lattmann T, Luscher TF, Shaw $S$. Obesity is associated with tissue-specific activation of renal angiotensin-converting enzyme in vivo: evidence for a regulatory role of endothelin. Hypertension 2000; 35: 329-336
18 Traupe T, D'Uscio LV, Muenter K, Morawietz H, Vetter W, Barton M. Effects of obesity on endothelium-dependent reactivity during acute nitric oxide synthase inhibition: modulatory role of endothelin. Clin Sci (Lond) 2002; 103 (Suppl 1): 13S-15S

19 Adiarto S, Emoto N, Iwasa N, Yokoyama M. Obesity-induced upregulation of myocardial endothelin-1 expression is mediated by leptin. Biochem Biophys Res Commun 2007; 353: 623-627

20 Tousoulis $D$, Charakida $M$, Stefanadis $C$. Inflammation and endothelial dysfunction as therapeutic targets in patients with heart failure. Int J Cardiol 2005; 100: 347-353

21 Parrinello G, Scaglione R, Pinto A, Corrao S, Cecala M, Di Silvestre G Amato P, Licata A, Licata G. Central obesity and hypertension: the role of plasma endothelin. Am J Hypertens 1996; 9: 1186-1191

22 Morawietz H, Goettsch W, Szibor M, Barton M, Shaw S, Hakim K, Zerkowski HR, Holtz J. Angiotensin-converting enzyme inhibitor therapy prevents upregulation of endothelin-converting enzyme-1 in failing human myocardium. Biochem Biophys Res Commun 2002; 295: 1057-1061

23 Ellinghaus $P$, Scheubel RJ, Dobrev D, Ravens U, Holtz J, Huetter J, Nielsch $U$, Morawietz $H$. Comparing the global mRNA expression profile of human atrial and ventricular myocardium with high-density oligonucleotide arrays. J Thorac Cardiovasc Surg 2005; 129: 1383-1390

24 Morawietz H, Szibor M, Goettsch W, Bartling B, Barton M, Shaw S, Koerfer R, Zerkowski HR, Holtz J. Deloading of the left ventricle by ventricular assist device normalizes increased expression of endothelin ETA receptors but not endothelin-converting enzyme-1 in patients with end-stage heart failure. Circulation 2000; 102: III188-III193

25 Sedmera D, Harris BS, Grant E, Zhang N, Jourdan J, Kurkova D, Gourdie $R G$. Cardiac expression patterns of endothelin-converting enzyme (ECE): implications for conduction system development. Dev Dyn 2008; 237: 1746-1753

26 Vignon-Zellweger N, Rahnenfuhrer J, Theuring F, Hocher B. Analysis of cardiac and renal endothelin receptors by in situ hybridization in mice. Clin Lab 2012; 58: 939-949

27 Fareh J, Touyz RM, Schiffrin EL, Thibault G. Endothelin-1 and angiotensin II receptors in cells from rat hypertrophied heart. Receptor regulation and intracellular $\mathrm{Ca}^{2+}$ modulation. Circ Res 1996; 78: 302-311

28 Niemann B, Rohrbach S, Catar RA, Muller G, Barton M, Morawietz H. Native and oxidized low-density lipoproteins stimulate endothelinconverting enzyme-1 expression in human endothelial cells. Biochem Biophys Res Commun 2005; 334: 747-753

29 Muller G, Catar RA, Niemann B, Barton M, Knels L, Wendel M, Morawietz $H$. Upregulation of endothelin receptor B in human endothelial cells by low-density lipoproteins. Exp Biol Med (Maywood) 2006; 231: 766-771

30 Morawietz H, Bornstein SR. Spironolactone for heart failure with preserved ejection fraction. N Engl J Med 2014; 371: 181 